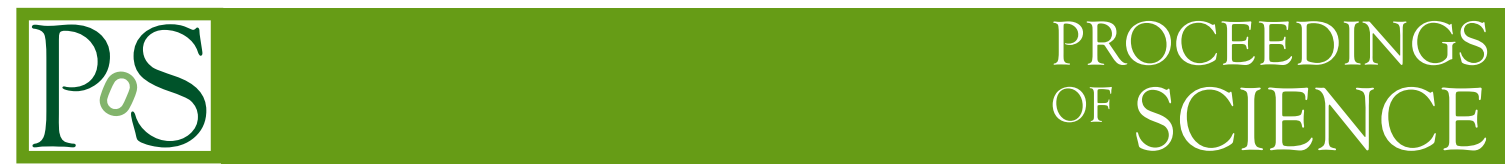

\title{
An update on the status of NSPT computations
}

\section{Michele Brambilla*}

Università di Parma and INFN

E-mail: michele.brambillaefis.unipr.it

\section{Francesco Di Renzo}

Università di Parma and INFN

E-mail: francesco.direnzodfis.unipr.it

We will discuss final results obtained by the Parma group for the computation of renormalization constants of quark bilinears for the regularizations defined by $\mathrm{nf}=2 \mathrm{Wilson}$ fermions/tree level Symanzik improved gauge and $\mathrm{nf}=4$ Wilson fermions/Iwasaki improved gauge actions. Computation has been performed in the framework of Numerical Stochastic Perturbation Theory (NSPT) up to three loop. Perturbative results will be compared with the ones coming from non perturbative determinations.

The 32nd International Symposium on Lattice Field Theory, 23-28 June, 2014

Columbia University New York, $N Y$

\footnotetext{
* Speaker.
} 


\section{Motivations}

Since its introduction non-perturbative computation [1] of Renormalization Constants (RC's) has been the preferred choice. Though, since quark bilinears are either finite or logarithmically divergent, nothing prevents the computation of RC's in Perturbation Theory (PT). From a practical point of view difficulties show up. Perturbative computations are affected by truncation errors; moreover, the perturbative series are badly convergent. In order to deal with these issues we put forward the computation up to three-loop [2, 3]. Such a computation requires the evaluation of a huge number of Feynman diagrams, making it in practice impossible. In order to evaluate all the contribution (order by order) we make use of Numerical Stochastic Perturbation Theory [ $\sharp$.

\section{RI'-MOM scheme and logarithmic divergences}

In the computation of RC's we have to deal with logarithmic divergences: while disentangling logarithmic and finite terms is in principle feasible, this would require a terrific numerical precision. We make use of an interesting feature of Regulator Independent (RI) renormalization scheme: in a perturbative computation of lattice RC's, logarithmic contributions can be inferred from continuum computations. To this extent we adhere to RI'-MOM scheme, which is one of the most widely used schemes on the lattice. Making use of continuum results we are able to subtract logarithmic contributions from our data so that we are only left with the computation of finite parts.

The procedure is the following. Being $S(p)$ the quark propagator and

$$
Z_{q}(\mu, \alpha)=-\left.i \frac{1}{12} \frac{\operatorname{Tr}\left(\not p S^{-1}(p)\right)}{p^{2}}\right|_{p^{2}=\mu^{2}}
$$

the definition of quark field RC, we constructs Green functions as expectation values computed on external quark states at fixed momentum $p$ :

$$
G_{\Gamma}(p)=\int d x\langle p|\bar{\psi}(x) \Gamma \psi(x)| p\rangle .
$$

We compute vertex functions by amputation, project on the tree-level structure

$$
\Gamma_{\Gamma}(p)=S^{-1}(p) G_{\Gamma}(p) S^{-1}(p) \quad O_{\Gamma}(p)=\operatorname{Tr}\left(\hat{P}_{O_{\Gamma}} \Gamma_{\Gamma}(p)\right)
$$

and finally impose the renormalization conditions

$$
\left.Z_{\Gamma}(\mu, \alpha) Z_{q}^{-1}(\mu, \alpha) O_{\Gamma}(p)\right|_{p^{2}=\mu^{2}}=1
$$

Next step is the subtraction of logarithms. A typical RC reads (the expansion is in the renormalized coupling)

$$
Z(\mu, \alpha)=1+\sum_{n>0} d_{n}(l) \alpha(\mu)^{n} \quad d_{n}(l)=\sum_{i=0}^{n} d_{n}^{(i)} l^{i} \quad l \equiv \log (\mu a)^{2} .
$$

From the definition of anomalous dimension

$$
\frac{1}{2} \frac{d}{d l} \log Z=\gamma=\sum_{n>0} \gamma_{n} \alpha(\mu)^{n}
$$

(where the last equality holds in PT) we can express the $d_{n}^{(i>0)}$ in terms of $\gamma_{m<n}$ and the coefficients of the $\beta$ function only. Here enters the tricky point: in a RI scheme we can read them from the continuum computations. 


\section{Zero quark mass}

Since we are interested in a mass-independent scheme, everything in sec. 目 is defined at zero quark mass. In perturbation theory this is simple: it only requires to plug the proper counterterms and doesn't need any chiral extrapolation (this is an advantage of PT, systematic errors due to chiral extrapolation are not involved).

Given the inverse quark propagator

$$
\hat{S}\left(\hat{p}, \hat{m}_{c r}, \beta^{-1}\right)^{-1}=i \hat{p}+\hat{m}_{W}(\hat{p})-\hat{\Sigma}\left(\hat{p}, \hat{m}_{c r}, \beta^{-1}\right)
$$

the critical mass is computed as the zero momentum contribution of the diagonal part of the selfenergy

$$
\left.\frac{1}{4} \operatorname{Tr} \hat{\Sigma}\left(\hat{p}, \hat{m}_{c r}, \beta^{-1}\right)\right|_{\hat{p} \rightarrow 0}=\hat{m}_{c r}
$$

Computing RC's up to three-loops requires knowledge of two-loop critical mass that in our cases of interest is analytically known. Since computation of critical mass comes almost for free we used it as a test (up to two-loop), and we obtained the three-loop values (fig. 1(c)] as a byproduct:

$$
\hat{m}_{c r}^{(3), t l s}=-3.94(4) \quad \hat{m}_{c r}^{(3), i w a}=-0.78(2)
$$

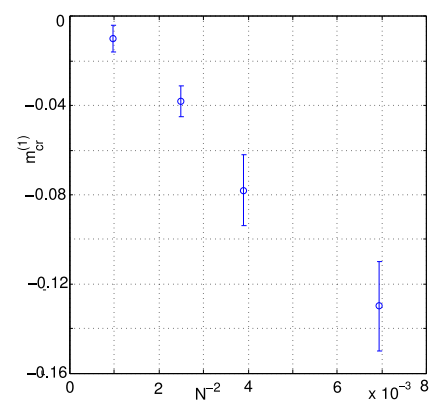

(a)

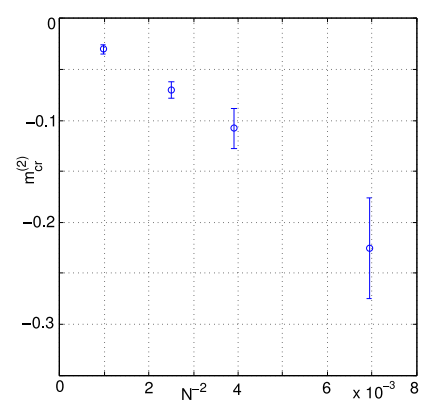

(b)

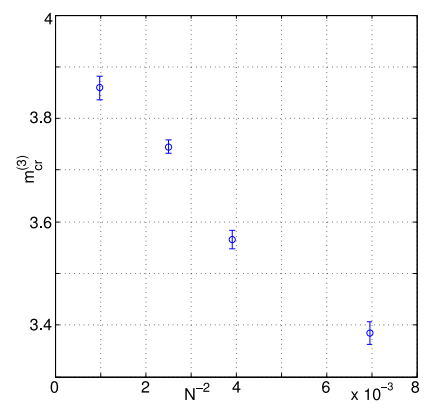

(c)

Figure 1: Critical mass for $n f=2$ Wilson fermions/tree level Symanzik improved gauge action in function of the inverse lattice size. One- (a) and two-loop (b) counterterms are already plugged in: this results in zero critical mass. We obtained three-loop (c) from our computations.

\section{Lattice artifacts}

As in any lattice computation we expect finite lattice spacing and finite volume effects. The former vanish in the $a \rightarrow 0$ limit, the latter can be expected (on dimensional grounds) to result in a $p L$ dependance $(N=L / a$ being the lattice size). Since we want to compute the RC's in both continuum and infinite volume, we need to take the two limits.

On a fixed volume $a \rightarrow 0$ limit can be obtained by computing the quantities for different momenta $\hat{p}$ and fitting the results in terms of hypercubic invariants. The possible terms are dictated by the symmetries of the specific observable. 
(a) tree-level Symanzik gauge action $/ \mathrm{n}_{f}=2$ Wilson fermion

\begin{tabular}{|c|c|c|c|c|}
\hline & $\begin{array}{c}\text { analytical } \\
\text { one-loop }\end{array}$ & one-loop & two-loop & three-loop \\
\hline \hline$Z_{S}$ & -0.6893 & $-0.683(7)$ & $-0.777(24)$ & $-1.96(14)$ \\
$Z_{P}$ & -1.1010 & $-1.098(11)$ & $-1.299(38)$ & $-3.19(21)$ \\
$Z_{V}$ & -0.8411 & $-0.838(6)$ & $-0.891(17)$ & $-1.870(65)$ \\
$Z_{A}$ & -0.6352 & $-0.633(4)$ & $-0.611(16)$ & $-1.198(57)$ \\
\hline \hline
\end{tabular}

(b) Iwasaki gauge action $/ \mathrm{n}_{f}=4$ Wilson fermion

\begin{tabular}{|c|c|c|c|c|}
\hline & $\begin{array}{c}\text { analytical } \\
\text { one-loop }\end{array}$ & one-loop & two-loop & three-loop \\
\hline \hline$Z_{S}$ & -0.4488 & $-0.442(6)$ & $-0.170(11)$ & $-0.33(11)$ \\
$Z_{P}$ & -0.7433 & $-0.739(7)$ & $-0.202(13)$ & $-0.58(11)$ \\
$Z_{V}$ & -0.5623 & $-0.561(7)$ & $-0.067(12)$ & $-0.367(61)$ \\
$Z_{A}$ & -0.4150 & $-0.419(6)$ & $-0.033(12)$ & $-0.236(56)$ \\
\hline \hline
\end{tabular}

Table 1: One-, two- and three-loop coefficients of the renormalization constants for quark bilinears. Expansions are in $\beta^{1}$. One-loop analytical results are reported for comparison.

By computing the observables on different volumes we can define finite volume corrections according to

$$
\begin{aligned}
\widehat{O}_{\Gamma}(\hat{p}, p L, v) & =\widehat{O}_{\Gamma}(\hat{p}, \infty, v)+\left(\widehat{O}_{\Gamma}(\hat{p}, p L, v)-\widehat{O}_{\Gamma}(\hat{p}, \infty, v)\right) \\
& \equiv \widehat{O}_{\Gamma}(\hat{p}, \infty, v)+\Delta \widehat{O}_{\Gamma}(\hat{p}, p L, v) \\
& \simeq \widehat{O}_{\Gamma}(\hat{p}, \infty, v)+\Delta \widehat{O}_{\Gamma}(p L)
\end{aligned}
$$

The rationale for the approximation is that we neglect corrections on top of corrections. All in all, a prototypal fitting form of ours reads

$$
\widehat{O}_{\Gamma}(\hat{p}, p L, v)=c_{1}+c_{2} \sum_{\sigma} \hat{p}_{\sigma}^{2}+c_{3} \frac{\sum_{\sigma} \hat{p}_{\sigma}^{4}}{\sum_{\rho} \hat{p}_{\rho}^{2}}+c_{4} \hat{p}_{v}^{2}+\Delta \widehat{O}_{\Gamma}(p L)+\mathscr{O}\left(a^{4}\right) .
$$

This approach requires a number of assumptions, therefore the effectiveness of the fit has to be assessed a posteriori. At one-loop we compared with analytical results; at higher orders we could assess stability of the procedure changing the order of expansions in $\hat{p}$, the interval of momenta considered and the number of different volumes.

\section{Results and discussion}

Tab. 1 shows the results of the computation. Errors are dominated by the stability of fits with respect to the change of fitting range, order of hypercubic expansion and number of lattice sizes simultaneously taken into account. As a confirm of the effectiveness of our method one-loop results agree with the previous analytical computations within errors. Tab. 1(a) and 1(b) show comparison with non-perturbative computations [5, 6] provided we sum the series at the proper $\beta$ value. Quoted 
(a) tree-level Symanzik gauge action $/ \mathrm{n}_{f}=2$ Wilson fermion

\begin{tabular}{|l|l|l|l|l|}
\hline & $Z_{V}$ & $Z_{A}$ & $Z_{S}$ & $Z_{P}$ \\
\hline \hline NSPT & $0.710(2)(28)$ & $0.788(2)(18)$ & $0.753(4)(30)$ & $0.601(5)(48)$ \\
ETMC(M1) & $0.659(4)$ & $0.772(6)$ & $0.645(6)$ & $0.440(6)$ \\
ETMC(M2) & $0.662(3)$ & $0.758(4)$ & $0.678(4)$ & $0.480(4)$ \\
\hline \hline
\end{tabular}

(b) Iwasaki gauge action $/ \mathrm{n}_{f}=4$ Wilson fermion

\begin{tabular}{|l|l|l|l|l|}
\hline & $Z_{V}$ & $Z_{A}$ & $Z_{S}$ & $Z_{P}$ \\
\hline \hline NSPT & $0.677(9)(39)$ & $0.769(9)(25)$ & $0.712(14)(36)$ & $0.538(15)(63)$ \\
ETMC(M1) & $0.655(03)$ & $0.762(04)$ & $0.649(5)$ & $0.478(2)$ \\
ETMC(M2) & $0.657(02)$ & $0.752(02)$ & $0.695(3)$ & $0.505(2)$ \\
\hline \hline
\end{tabular}

Table 2: Summation of the series at $\beta=4.05$ (tree-level Symanzik) and $\beta=2.10$ (Iwasaki) and comparison with results from [5] and [6] respectively.

errors are respectively statistical errors and a rough estimate of truncation errors, where the latter are simply taken as the highest order contribution.

Observe that the truncation errors are quite large. This is due to the fact that the relative weight of three-loop contribution is large with respect one- and two-loop. We can spot a fair agreement in the Iwasaki case between perturbative and non-perturbative results even considering the statistical error only. One the other hand there is a large deviation in the Symanzik case, more manifest in the divergent quantities. Notice that in the case of divergent quantities the different prescriptions "M1" and "M2" used in the non-perturbative case for dealing with irrelevant effects give results not in full agreement.

Before we proceed with the analysis of the summations let's inspect the behavior of finite lattice spacing effects. We can assess irrelevant effects by discarding the continuum limit ( $c_{1}$ in 4.1 ) and finite size contributions $\Delta \widehat{O}_{\Gamma}(p L)$ :

$$
\tilde{O}_{\Gamma}^{(i)}(\hat{p}, v)=c_{2}^{(i)} \sum_{\sigma} \hat{p}_{\sigma}^{2}+c_{3}^{(i)} \frac{\sum_{\sigma} \hat{p}_{\sigma}^{4}}{\sum_{\rho} \hat{p}_{\rho}^{2}}+c_{4}^{(i)} \hat{p}_{v}^{2}+\mathscr{O}\left(a^{4}\right) .
$$

The resummed quantity

$$
\sum_{i=1}^{3} \beta^{-i} \frac{1}{4} \sum_{v=1}^{4} \tilde{O}_{\Gamma}^{(i)}(\hat{p}, v)
$$

can be regarded as the irrelevant contributions to $Z_{\Gamma}$ (2.1). The different impact of (continuum-like) rotational symmetry in the two cases is shown in fig. 国. Observe that the amount of violation is not decided by the choice of momenta.

We can try to better understand the convergence proprieties of the series considering expansions in different couplings. This technique is usually referred to as Boosted Perturbation Theory [7]. We will consider the following couplings ( $P$ is the $1 \times 1$ plaquette):

$$
x_{0}=\beta^{-1} \quad x_{1}=\frac{\beta^{-1}}{\sqrt{P}} \quad x_{2}=-\frac{1}{P^{(0)}} \log (P) \quad x_{3}=\frac{\beta^{-1}}{P} .
$$

Switching from $x_{0}$ to $x_{1}, x_{2}$ and finally to $x_{3}$ the values of the coupling are getting larger and larger. A remark before proceeding. We do not have non-perturbative simulations in the same setting we 


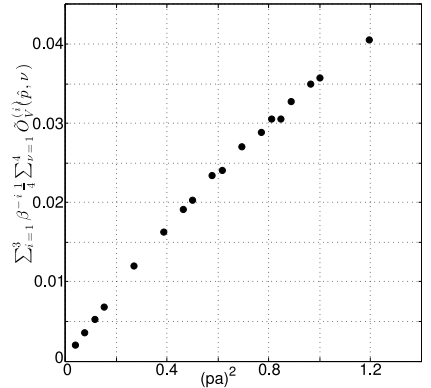

(a) tree-level Symanzik gauge action $/ \mathrm{n}_{f}=2$ Wilson fermions

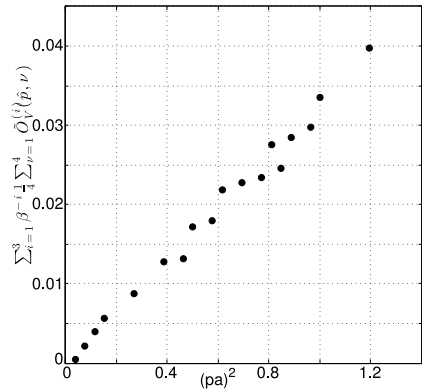

(b) Iwasaki gauge action $/ \mathrm{n}_{f}=4$ Wilson fermions

Figure 2: Contribution of irrelevant effects to $Z_{V}$ for the two different actions.

are dealing with. In spite of this we estimate the value of $P$ summing the perturbative expansion of the plaquette and we take into account the relevant statistical and truncation errors in the final summation. We will consider only tree level Symanzik case, since Iwasaki results are already in good agreement.

\begin{tabular}{|c|c|c|c|c|c|c|}
\hline & $x_{0}$ & $x_{1}$ & $x_{2}$ & $x_{3}$ & $(\mathrm{M} 1)$ & $(\mathrm{M} 2)$ \\
\hline \hline$Z_{V}$ & $0.710(2)(28)$ & $0.686(21)$ & $0.688(17)$ & $0.661(55)$ & $0.659(4)$ & $0.662(3)$ \\
$Z_{A}$ & $0.788(2)(18)$ & $0.773(12)$ & $0.775(9)$ & $0.763(26)$ & $0.772(6)$ & $0.758(4)$ \\
$Z_{S}$ & $0.753(4)(30)$ & $0.727(29)$ & $0.726(27)$ & $0.705(49)$ & $0.645(6)$ & $0.678(4)$ \\
$Z_{P}$ & $0.601(5)(48)$ & $0.558(45)$ & $0.558(41)$ & $0.526(73)$ & $0.440(6)$ & $0.480(4)$ \\
\hline \hline
\end{tabular}

Table 3: Quark bilinears $R C$ 's for tree-level Symanzik improved gauge action at $\beta=4.05$ summed in different couplings. Non-perturbative results are reported for comparison.

In tab. B we can see that $Z_{V}$ and $Z_{A}$ get closer to the non-perturbative result. The central value agrees with non-perturbative results, while the error becomes large. Observing fig. 3(a) we can understand the reason: the series start fluctuating and two- and three-loop basically cancel each other.

Examining logarithmically divergent currents the situation is different: after the boosting procedure there are still discrepancies between summations and non-perturbative results. Notice that (fig. 3(b) ) using the coupling $x_{3}$ the series already started reshuffling: we cannot improve the situation by using a larger coupling. Even though there is of course the possibility that higher order terms should be included, another issue has to be considered. The different approach "M1" and "M2" brings to different results. Thus there is the possibility that given the interplay between IR and UV effects, non-perturbative computations suffer from finite-volume effects.

\section{Conclusion}

We presented the final results for the computation of RC's of quark bilinears for the regularizations defined by $\mathrm{nf}=2$ Wilson fermions/tree level Symanzik improved gauge [2] and $\mathrm{nf}=4$ Wilson fermions/Iwasaki improved gauge [B]. While there is no constraint on computing finite constants 


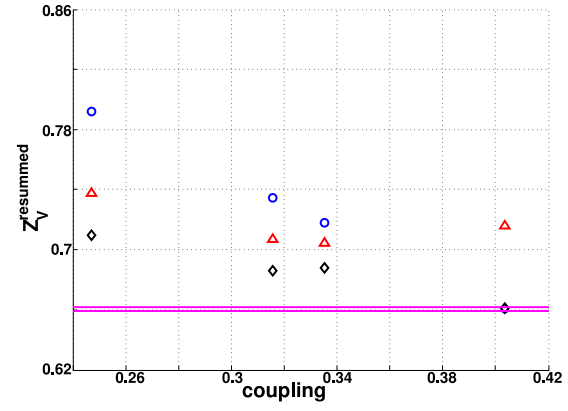

(a)

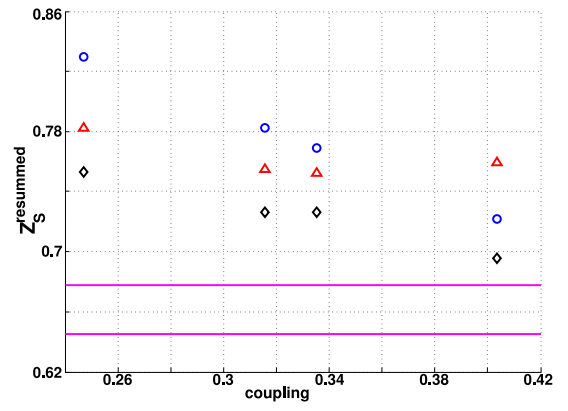

(b)

Figure 3: Summation of the series at one- (blue circles), two- (red triangles) and three-loop (black diamonds) at different couplings (from left to right $x_{0}, x_{1}, x_{2}$ and $x_{3}$ ). At the large coupling $x_{3}$ series summation reshuffle.

we are limited in the divergent ones because continuum computations are available at three-loop order in the RI- MOM scheme.

NSPT provide an independent approach with respect to non-perturbative computations, with different systematic effects. High loop computations can provide a new handle to correct nonperturbative computations with respect to irrelevant contributions. We proposed a method for the assessment of finite size effects that can be useful in the non-perturbative case as well.

We are grateful to the Research Center for Nuclear Physics and to the Cybermedia Center in Osaka University for the time that was made available to us on their computing facilities RCNP and CMC. We also acknowledge computer time on the Tramontana I.N.F.N. facility and on the Aurora system. We thank the AuroraScience Collaboration for the latter.

\section{References}

[1] G. Martinelli, C. Pittori, C. T. Sachrajda, M. Testa and A. Vladikas, A General method for nonperturbative renormalization of lattice operators, Nucl. Phys. B 445, 81 (1995).

[2] M. Brambilla and F. Di Renzo, High-loop perturbative renormalization constants for Lattice QCD (II): three-loop quark currents for tree-level Symanzik improved gauge action and $n_{f}=2$ Wilson fermions, Eur. Phys. J. C 73, 2666 (2013).

[3] M. Brambilla, F. Di Renzo and M. Hasegawa, High-loop perturbative renormalization constants for Lattice QCD (III): three-loop quark currents for Iwasaki gauge action and $n_{f}=4$ Wilson fermions, Eur. Phys. J. C 74, 2944 (2014).

[4] F. Di Renzo and L. Scorzato, Numerical Stochastic Perturbation Theory for full QCD, JHEP 04 (2004) 073.

[5] M. Constantinou et al. [ETM Collaboration], Non-perturbative renormalization of quark bilinear operators with $N_{f}=2$ (tmQCD) Wilson fermions and the tree-level improved gauge action, JHEP 1008, 068 (2010).

[6] N. Carrasco et al. [ETM Collaboration], Up, down, strange and charm quark masses with $N_{f}=$ 2+1+1 twisted mass lattice QCD, Nucl. Phys. B 887 (2014) 19.

[7] G. P. Lepage and P. B. Mackenzie, On the viability of lattice perturbation theory, Phys. Rev. D 48 , 2250 (1993). 